\title{
Twist Springback Measurement of Autonomous Underwater Vehicle Propeller Blade Based on Profile Deviation
}

\author{
${ }^{1,2}$ Ahmad Baharuddin Abdullah, ${ }^{2}$ Mohd Sapuan Salit, \\ ${ }^{1}$ Zahurin Samad, ${ }^{1}$ Khaleed Hussain MTandoor and ${ }^{2}$ Nuraini Abdul Aziz \\ ${ }^{1}$ School of Mechanical Engineering, \\ Universiti Sains Malaysia, Engineering Campus, 14300 Nibong Tebal, Penang, Malaysia \\ ${ }^{2}$ Department of Mechanical and Manufacturing Engineering, \\ Faculty of Engineering, University Putra Malaysia, 43400 Serdang, Selangor, Malaysia
}

Received 2012-07-30, Revised 2013-05-29; Accepted 2013-05-31

\begin{abstract}
Geometrical defects that are attributable to springback are among the major defects induced by the sheet metal forming process. Such defects are critical for sections with varying thickness, such as Autonomous Underwater Vehicle (AUV) propeller blades. In this study, the springback in the twist-bending of an AA6061 propeller blade was quantified by comparing the targeted and manufactured profiles obtained using the commercial threedimensional surface measurement technique. The results show that the twist springback becomes larger as the twist angle increases. Similarly, the twist springback increases with increasing deformation ratio.
\end{abstract}

Keywords: Twist Springback, Propeller Blade, Cold Forging, Profile Deviation

\section{INTRODUCTION}

The formation of twist bending is one of the primary sheet-forming processes currently employed by major automotive industries. However, the process results in a severe springback problem, which affects the accuracy of the finished part. Although springback is considered a manufacturing error, its proper assessment and precise control has been a rather challenging task (Lange, 1985). A large number of studies have already been conducted on the springback problem, but none have focused on the twist springback that involves thickness changes or thinning. From a mechanics point of view, the twist springback results from torsional moments in the crosssection of the workpiece. The torsional displacement develops from the unbalanced elastic deformation and residual stresses acting in the part to create a torsional moment, which tends to rotate one end of the part relative to another. The torsional moment can originate from the in-plane residual stresses in the flange, in the sidewall, or both (Li et al., 2011). In the production of a propeller blade, bending twist forming is commonly used to produce the twisted shape of the blade. Basically, few experimental approaches can be used to measure the springback, as discussed by Abdullah et al. (2012a).

A propeller blade has a complex shape and typically comprises four parts, namely, the suction face, pressure face, leading edge and trailing edge (Hsu et al., 2006). In most cases, a Coordinate Measurement Machine (CMM) is employed to inspect a blade defect. Size and shape complexity limit the use of conventional methods, such as CMM. In addition, longer setup and measurement time are among the disadvantages of this method. Therefore, the improvement of the part inspection technique through integrated surface measurement and profile deviation methods is proposed in this study.

This study emphasizes the twist springback measurement of an Autonomous Underwater Vehicle (AUV) propeller blade manufactured using cold forging process. The measurement is based on the profile obtained from a three-Dimensional (3D) surface

Corresponding Author: Ahmad Baharuddin Abdullah, School of Mechanical Engineering, Universiti Sains Malaysia, Engineering Campus, 14300 Nibong Tebal, Penang, Malaysia 
measurement system called Infinite Alicona Focus. In this study, the twist springback was determined by comparing the obtained profiles of the blade and the punch, where the former is considered the manufactured part and the latter represents the targeted part. This study will begin with an introduction, followed by a discussion of the related work on the profile measurement approach and the basic design parameters considered in the design of a propeller. The proposed methodology is then presented, followed by the presentation of the results and discussion. The study ends with the conclusion.

\subsection{Related Work}

To date, relatively few methods and techniques have been developed to assess geometrical defects based on profile deviation. In most cases, Computed-Aided Design (CAD) models and the CMM have become the main sources of profile data. To improve measurement accuracy, Hsu et al. (2006) suggested a two-step measurement procedure, including a rough measurement and a fine measurement, along with the calculation of the back-off directions. In their approach, blade profile analysis involves several algorithms, including a best-fit algorithm, blade parameter computation and profile and tolerance zone analysis. Qiu et al. (2000) utilized a CMM facility to obtain data and then measured form deviation relative to the approximate contour. Similarly, Pahk and Ahn (1996), using an integrated $\mathrm{CAD} /$ computer-aided instruction system, analyzed the profile tolerance of parts, where the actual measured points are obtained by finding the closest points on the CAD geometry via the subdivision technique. For accurate profile tolerance, the Tschebyscheff norm was applied iteratively. In another case, Lam et al. (2002) developed a new precision caliper system at a lower cost. On the other hand, Li et al. (2010) proposed a method for the automatic ultrasonic inspection of defects in a propeller blade. The 3D surface data are obtained via ultrasonic measurement. Based on the C-scan image, the defective area can be detected.

Alternatively, the optical technique has become one of the prefered options because of flexibility and reliability. For example, (D'Acquisto and Fratini, 2001) developed a measurement technique based on the shadow moiré method to evaluate the springback phenomenon in deep-drawing operations. In another case, Abdullah et al. (2012b) evaluated the profile deviation of a propeller blade using a 3D surface measurement system. The profile is developed using a commercial system based on the focus-variation principle to determine the deviation. In most cases, the Finite Element Method (FEM) has been utilized to predict the springback. For example, Makem et al.
(2008) proposed a virtual inspection method for the measurement of twist angle deviation using FEM and CMM. In their approach, a number of points on the surface of the blade were selected and measurements of the twist angle error were made based of the profile deviation before and after forging.

Dejardin et al. (2010) measured the springback of a truncated cone based on a geometrical profile using experimental and finite element models. The profile was obtained by using the laser method while considering transverse sections. The springback was characterized through the cut ring method. Ambrogio et al. (2004) quantified the geometrical discrepancies in incremental forming, with emphasis on the elastic springback evaluation and compensation using a reverse engineering technique. In their work, profiles were obtained using a laser scanning system and the product geometry was then rebuilt based on the laser triangulation principle. The resultant profiles were then compared with the expected geometrical model. Chang et al. (2002) observed the geometrical profile error in the cold ironing of a gear to investigate the form errors attributed to die-elasticity and to the springback of the component shape.

\subsection{Design Parameters}

Numerous methods can be employed for propeller design. Figure 1 defines a number of terms that are used to describe propeller performance. The total radius $R$ of the propeller denotes the distance from the center to the tip. The chord length $c$ is the straight-line width of the propeller at a given distance along the radius. Depending on the design of the propeller, the chord length may be constant along the entire radius, or it may vary along the radius of the propeller. Another variable is the twist angle $\beta$ of the propeller, which may also vary along the radius of the propeller, as shown in Fig. 2.

Theoretically, the efficiency of the propeller can be determined by the ratio of velocity $(\mathrm{mm} / \mathrm{s})$ and thrust $(\mathrm{N})$ as an output to the power (W) as an input. Higher output will result in higher efficiency. Based on this principle, the performance of the propeller primarily depends on the thrust and the advance ratio. The thrust is directly related to the power input, whereas the advance ratio is influenced by the geometrical shape of the blade. Therefore, the twist angle may not achieve the targeted value because of error, thus resulting in lower propeller efficiency. The relationship between the twist angle $\beta$ and local radius $r$ can be determined using the Equation 1 given by (Habali and Saleh, 2000):

$\beta=\left(\left(\frac{R \alpha_{t}}{r}\right)-\alpha_{t}\right)-k\left(1-\frac{r}{R}\right)$ 


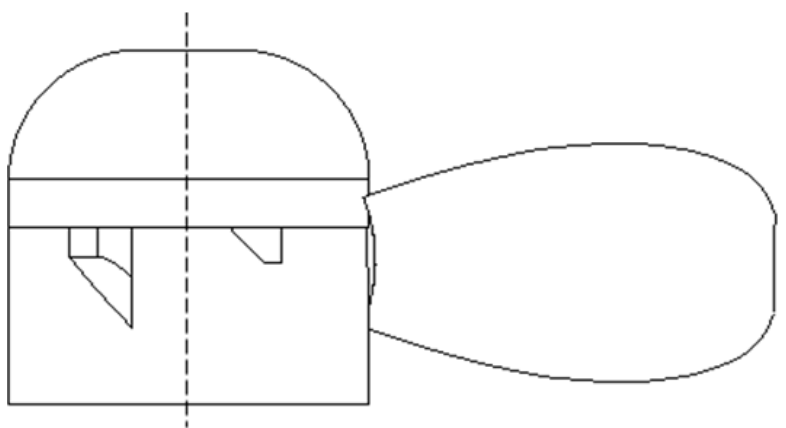

(a)

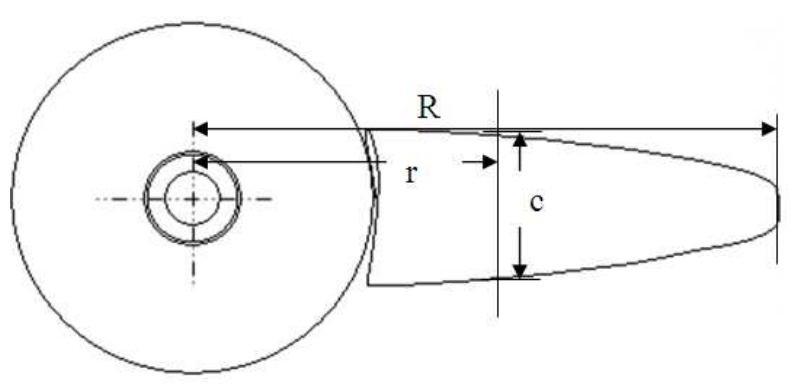

(b)

Fig. 1. Schematic drawing of the propeller configuration from (a) side and (b) top view

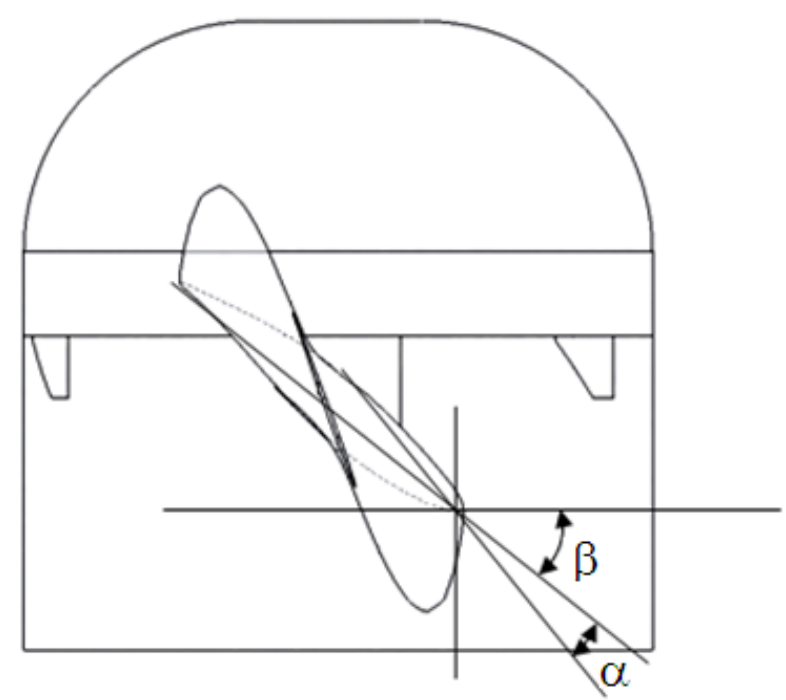

Fig. 2. Orientation of the blade and the twist angle definition

Where:
$\mathrm{R}=$ Total radius,
$\mathrm{r}$ = Local radius,
$\mathrm{A}=$ Angle of attack $=1.54^{\circ}$ (Ideal) and
$\mathrm{K}=$ Constant $(\mathrm{k}>0)$.

\section{MATERIALS AND METHODS}

\subsection{Definition of Springback and Deformation Ratio}

The springback is measured based on twist angle deviation and can be defined as $\beta_{\mathrm{b}}-\beta_{\mathrm{d}}$, where $\beta_{\mathrm{b}}$ is the twist angle of the blade and $\beta_{\mathrm{d}}$ is the twist angle measured on the punch. The twist angle $\beta$ is defined in terms of the chord line with respect to the horizontal line and blade twist angle defines the pitch settings at each position along the blade according to local flow conditions (Habali and Saleh, 2000), as demonstrated in the Fig. 3. Given that the twist angle varies along the blade, therefore for 2D investigation, the blade is cut into few sections, as shown in Fig. 4.

In this study, the effect of the deformation ratio on the thickness error pattern will be observed. In this work, the deformation Ratio $\mathrm{D}_{\mathrm{r}}$ is defined as $=\mathrm{Ln}$ $\left(t_{o} / t_{f}\right)$, where $t_{o}$ is the initial thickness and $t_{f}$ is the minimal thickness at final stage.

\subsection{Profile Mapping}

The profile was measured using a standard 3D surface measurement system called Alicona Infinite Focus (Alicona Imaging Ltd.), as shown in Fig. 5. The AUV propeller blade was placed on the table and a lens with $2.5 \times$ magnification (vertical resolution of $2300 \mathrm{~nm}$ ) was used to scan the profile.

\subsection{Profile Deviation Measurement}

The process begins with the filtering stage, where all undesired noises are deleted. The proposed doublemirroring technique is then applied. The mirroring is performed for each point that comprises the profile. Only one of the profiles, that is, either the punch profile or the blade profile, will be selected. The steps of the mirroring algorithm are given below and the implementation is shown in Fig. 6.

Determination of the midpoint:

$$
\left(\mathrm{m}_{\mathrm{x}}, \mathrm{m}_{\mathrm{y}}\right)=\text { Coordinate of half chord length }
$$

Computation of the vector of each point:

$$
\mathrm{d}_{\mathrm{x}}, \mathrm{d}_{\mathrm{y}}=\left(\mathrm{x}_{1}-\mathrm{m}_{\mathrm{x}}, \mathrm{y}_{1}-\mathrm{m}_{\mathrm{y}}\right)
$$

First rotation, $180^{\circ}$ from the midpoint

$$
\mathrm{d}_{\mathrm{x} 1}=-\mathrm{d}_{\mathrm{y}} \text { and } \mathrm{d}_{\mathrm{y} 1}=\mathrm{d}_{\mathrm{x}}
$$

Determination of new points

$$
\mathrm{x}_{2}=\mathrm{m}_{\mathrm{x}}+\mathrm{d}_{\mathrm{x} 1} \text { and } \mathrm{y}_{2}=\mathrm{m}_{\mathrm{y}}+\mathrm{d}_{\mathrm{y} 1}
$$


Ahmad Baharuddin Abdullah et al. / American Journal of Applied Sciences 10 (5): 515-524, 2013

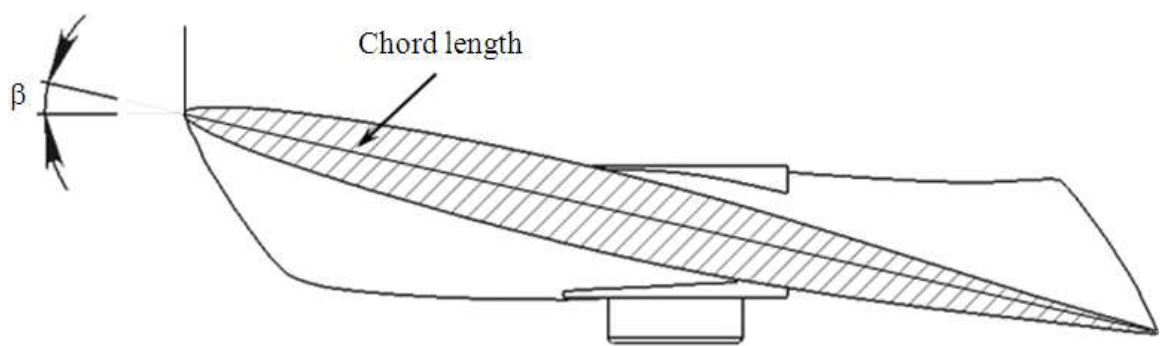

Fig. 3. The definition of the twist angle and chord length

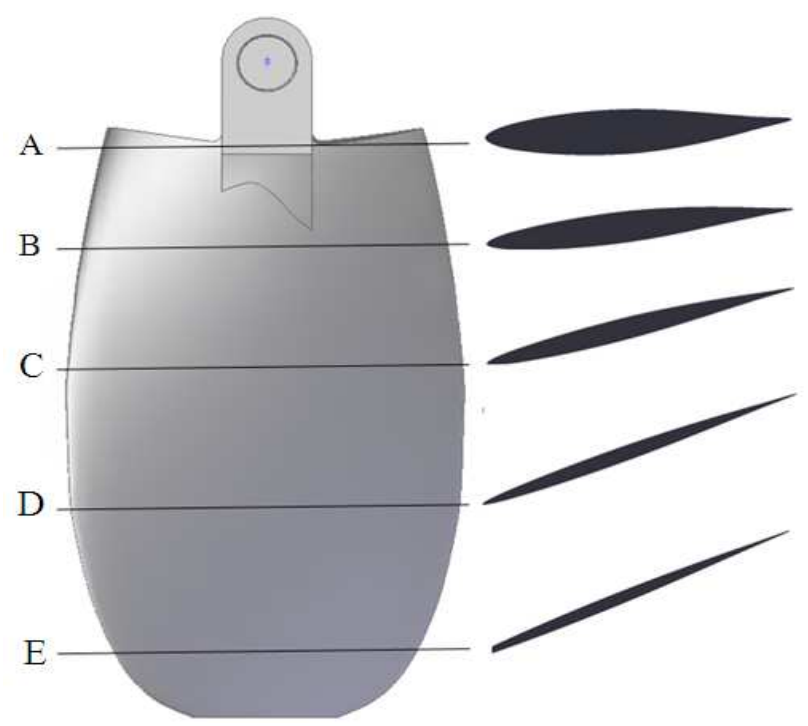

Fig. 4. Five sections of the blade

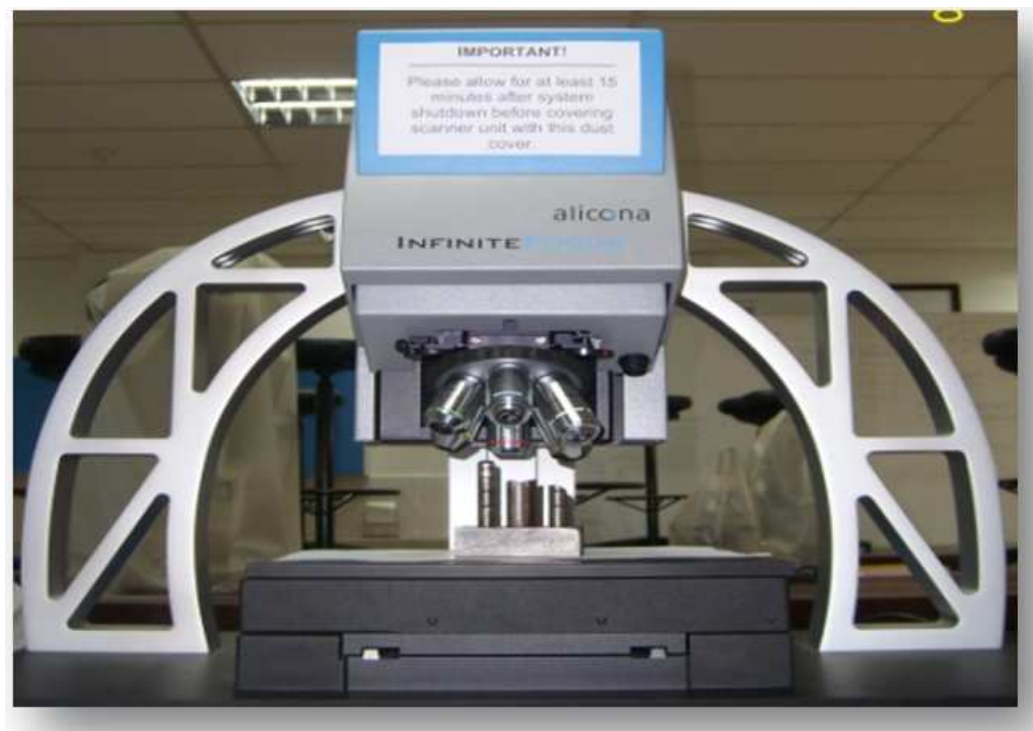

Fig. 5. The punch placed on the table for scanning 


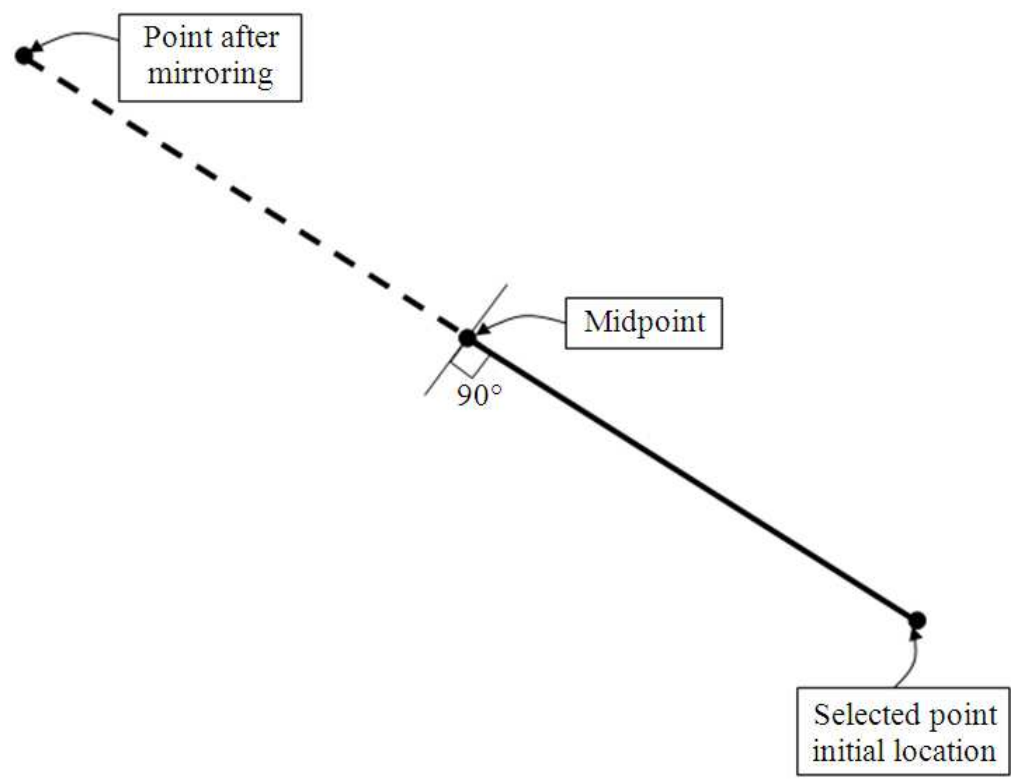

Fig. 6. The mirroring technique for one point

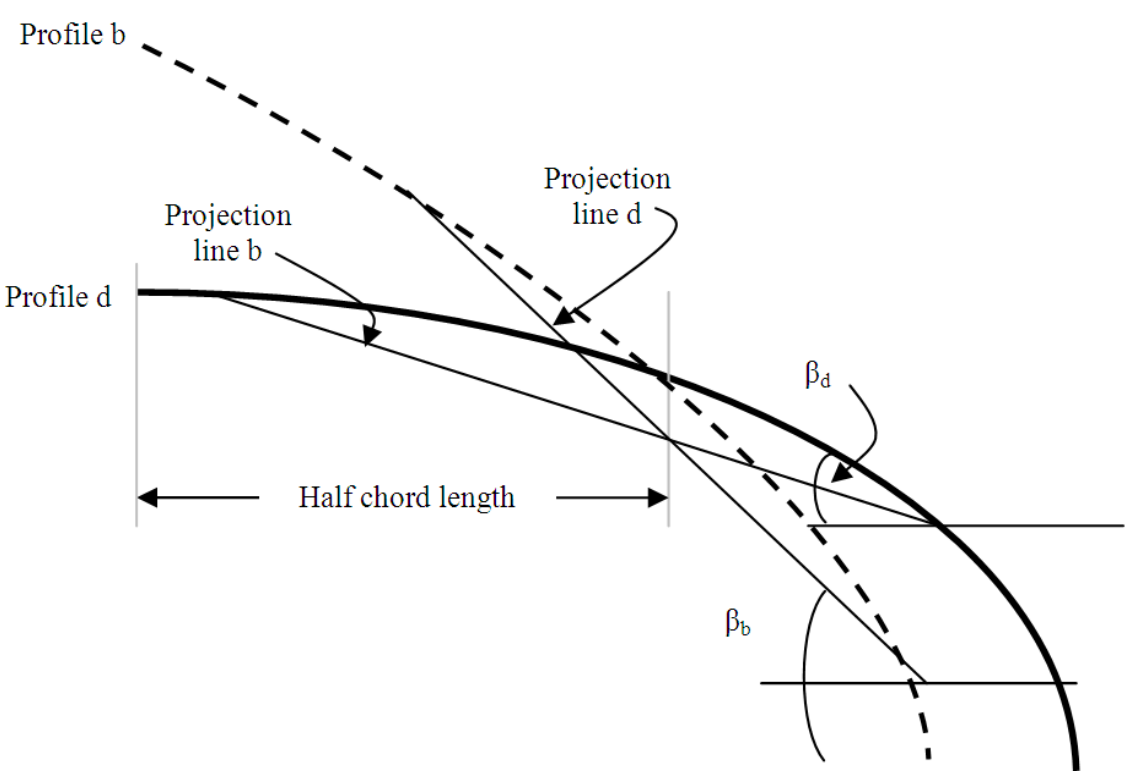

Fig. 7. The twist angle deviation measurement method

Repetition of the same steps for all points and sides.

After completion of the mirroring process, the deviation can be measured. In this approach, the intersection of both profiles must be equal to half of the chord length, while the mid-point is assumed to be fixed. A straight projection line can then be drawn between two points defined on the profile, as shown in
Fig. 7. The starting and end points of the line must be at the same distance from the midpoint. The angle of both profiles can then be determined. This approach has been initiated from the technique developed by Makem et al. (2008). Notably, both projection lines must be intersected at same the intersection location as the initial profiles. 


\section{RESULTS}

In this study, the profile of the punch is considered to be the targeted profile, whereas the profile of the blade is taken as the manufactured profile. To ensure better visualization, white acrylic spray was applied to the surface of the die and the punch. For benchmarking, the twist angle of the blade from the theoretical and nominal models was mapped, as shown in Fig. 8 and a very good agreement was observed.

Figure 9a shows the profile constructed by using the profile of the blade section obtained from Alicona after the filtering and cleaning processes. Figure 9b illustrates the profile obtained after the mirroring technique was applied. The measured twist springback is summarized in Table $\mathbf{1}$.

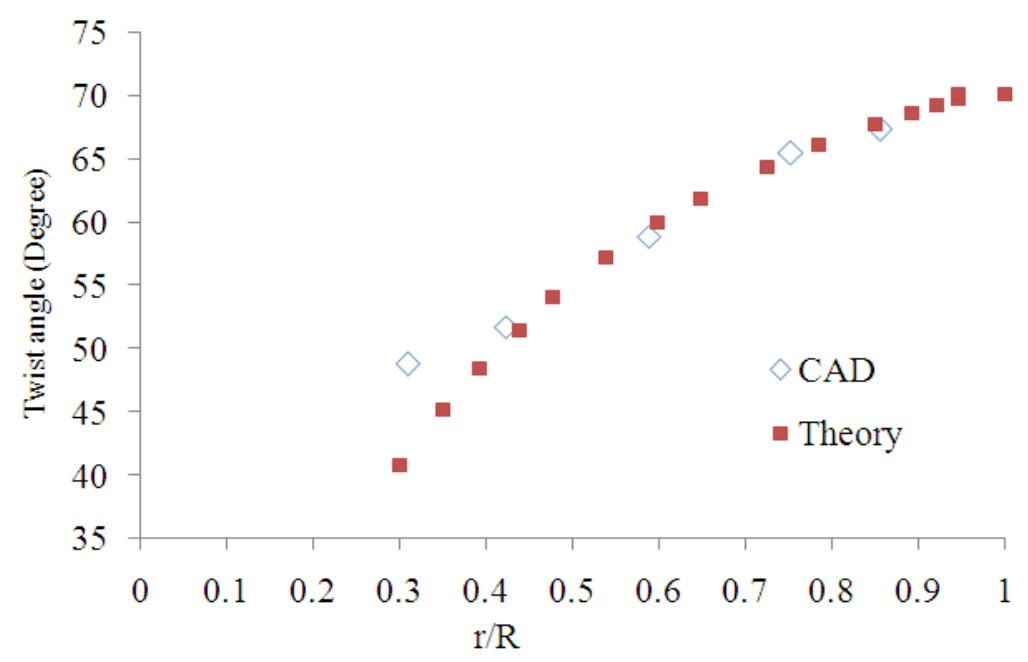

Fig. 8. The twist angle distribution

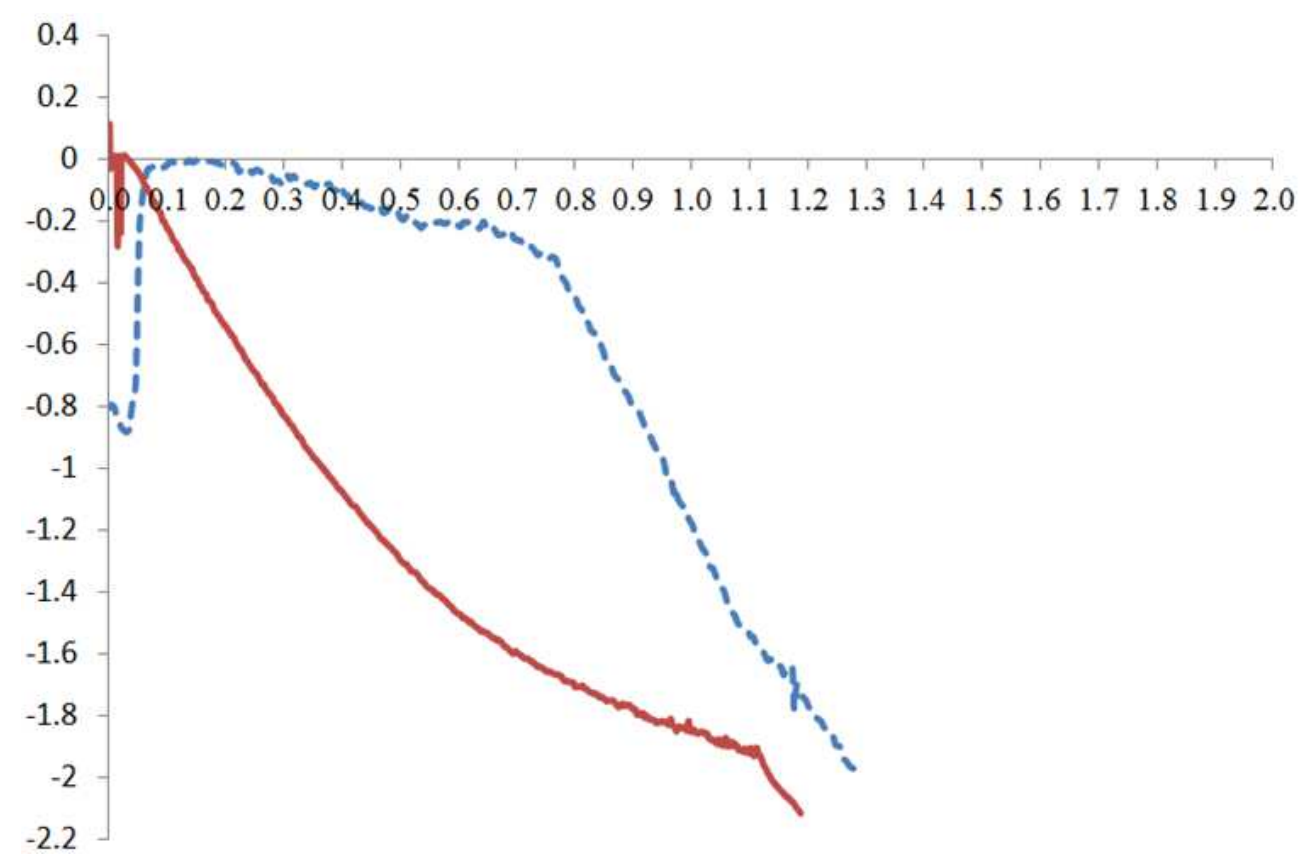

(a) 


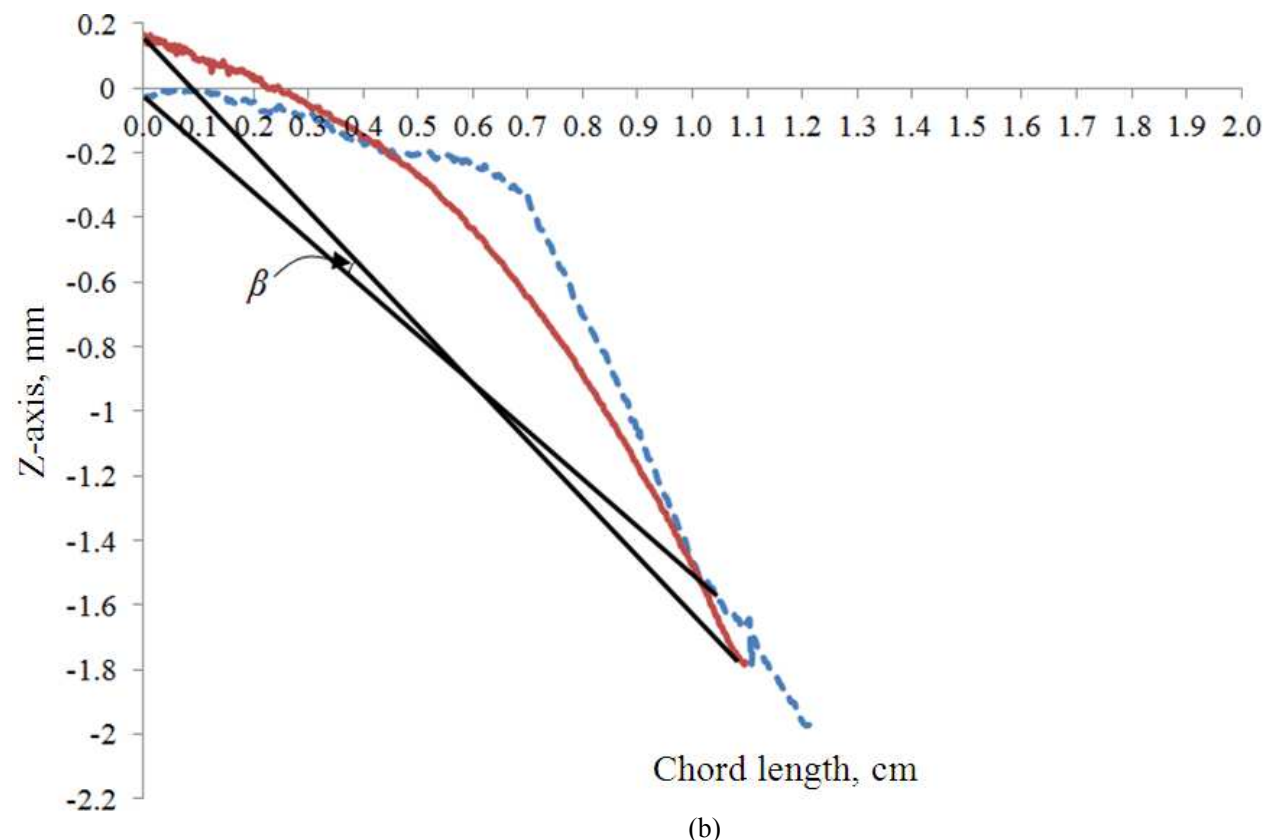

Fig. 9. The resulted profiles of the blade and punch (a) before and (b) after applying the algorithm

Table 1. Twist springback measured

\begin{tabular}{llllr}
\hline Section & Deformation Ratio & $\beta_{\mathrm{b}}$ (Degree) & $\beta_{\mathrm{d}}$ (Degree) & Springback (\%) \\
\hline A & 1.990 & 47.54 & 48.80 & 2.58 \\
B & 2.802 & 49.27 & 51.76 & 4.81 \\
C & 2.969 & 52.67 & 58.90 & 10.58 \\
D & 3.219 & 56.20 & 65.48 & 14.17 \\
E & 5.704 & 56.50 & 67.35 & 16.11 \\
\hline
\end{tabular}

\section{DISCUSSION}

The twist angle was measured based the edge of the profile by referring to the chord length of each section. The orientation of the blade is considered to be perpendicular to the hub assembly by taking the pin head as reference, as shown in Fig. 4. The results show different patterns, where the twist springback becomes larger as the twist angle increases toward the tip.

Based on the result obtained from the measurement, the effect of the deformation ratio is considered to be significant, as depicted in Fig. 10. As the deformation ratio increases, the springback also increases, especially at a deformation ratio of 3 . However, beyond this deformation ratio, the increments gradually decline because the twisting goes beyond the plastic region of the section.
Similarly, the springback pattern along the blade length exhibits the same trend, as shown in Fig. 11. This result agrees with that shown in Fig. 10. The springback becomes larger toward the tail of the blade as the twist angle increases and the deformation ratio increases as well. For a miniaturized blade with a complex shape, this problem may rapidly worsen, thus increasing the possibility of product failure.

For validation, a profile measurement using CMM was conducted. For this purpose, a Mitutoyo CrystaPlus M443 CMM was used. The accuracy and resolution of the machine are 4.0 and $0.5 \mu \mathrm{m}$, respectively. The profile of the punch was mapped on a worksheet and then compared with the profile obtained from the proposed approach, as shown in Fig. 12. As a result, the deviation is less than $10 \%$, which can be considered low for such springback cases. 
Ahmad Baharuddin Abdullah et al. / American Journal of Applied Sciences 10 (5): 515-524, 2013

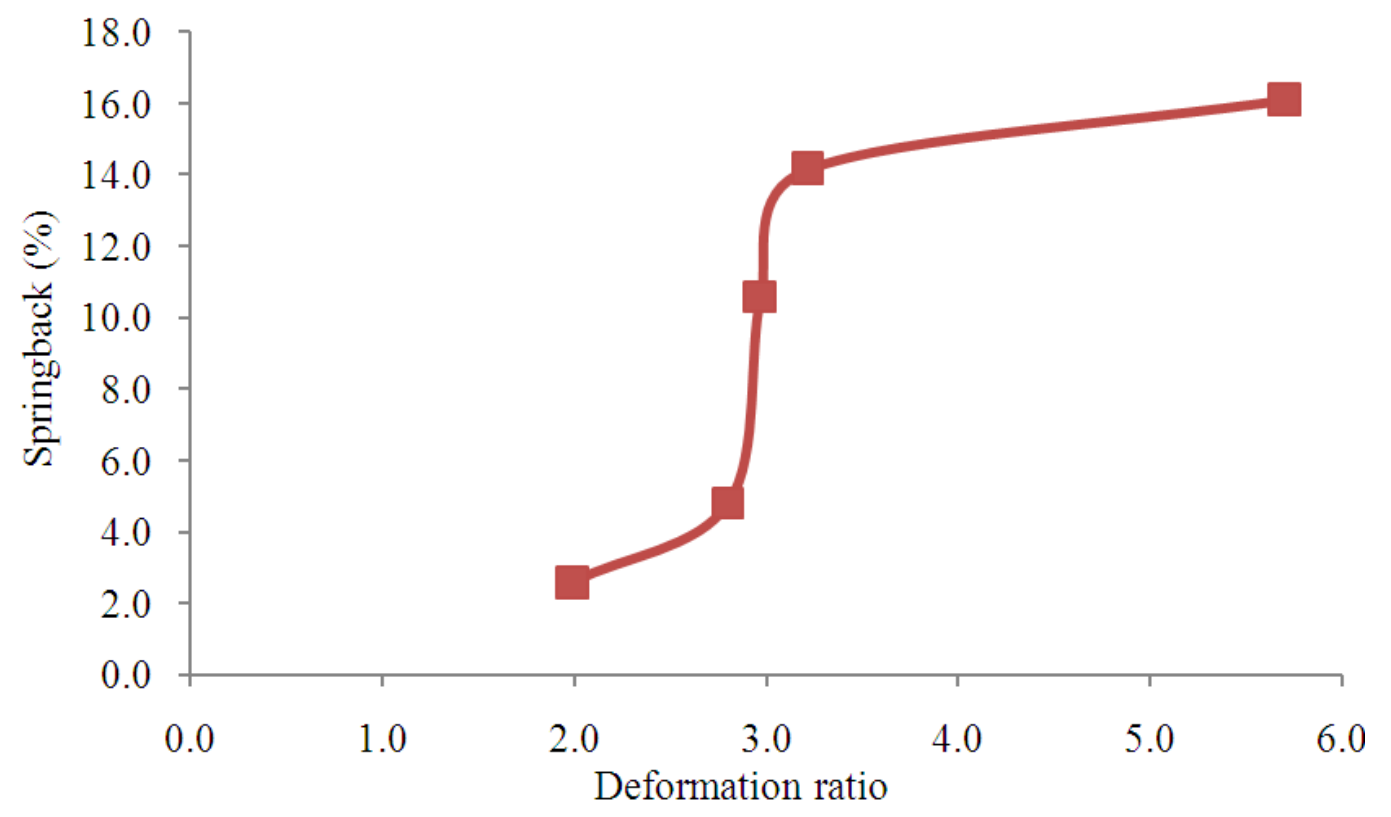

Fig. 10. The springback pattern and effect of deformation ratio

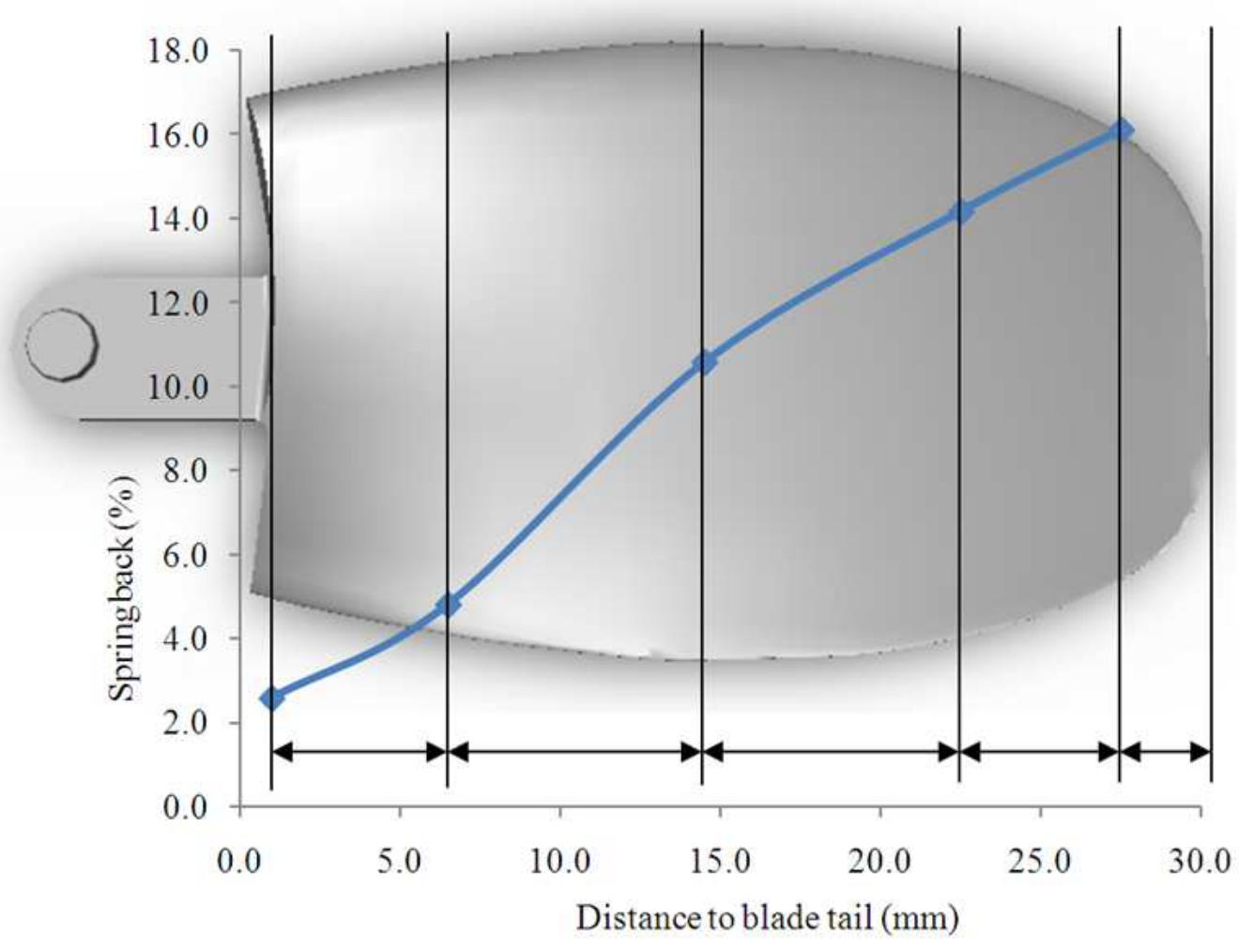

Fig. 11. The twist springback pattern along the blade length 


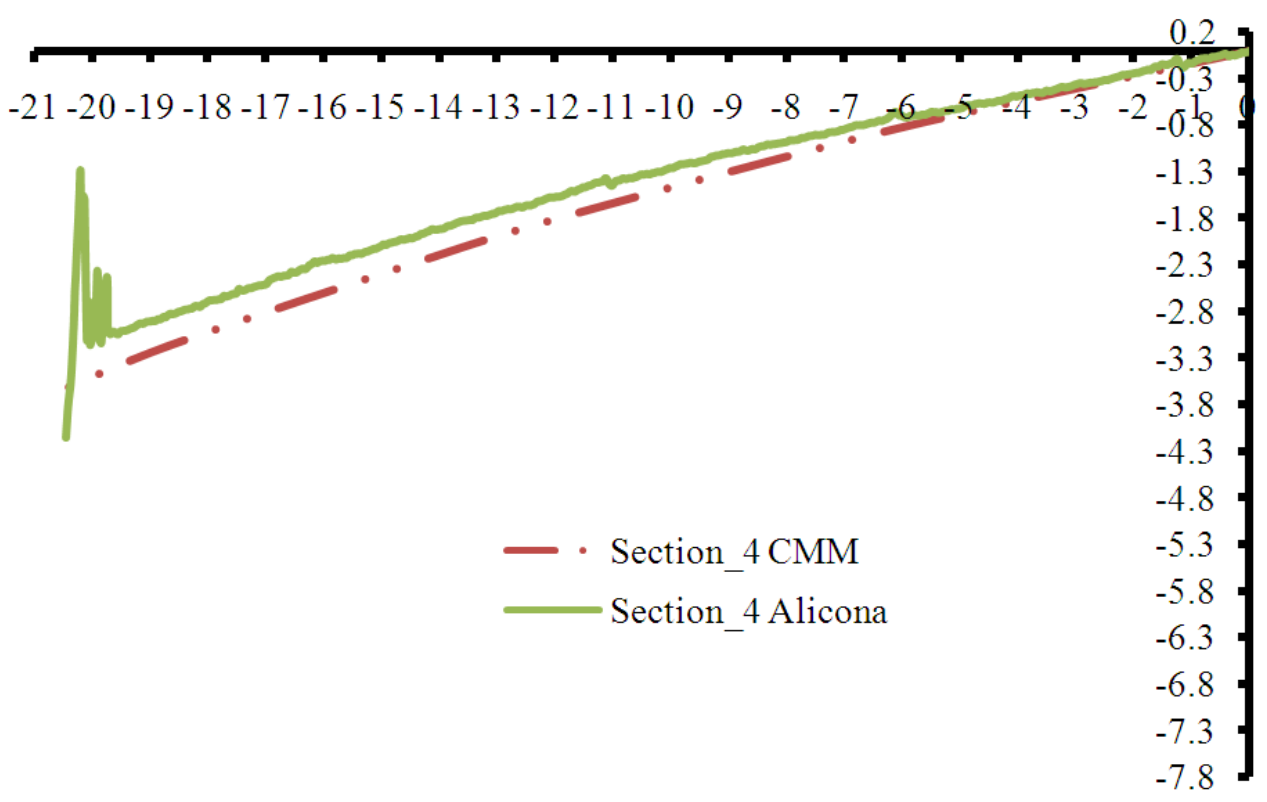

Fig. 12. The comparison between the profiles obtained from Alicona and the CMM

\section{CONCLUSION}

This study presented the measurement of twist springback of cold-forged AUV propeller blade. The measurements were based on the difference between the profile obtained from the $3 \mathrm{D}$ surface measurement technique, namely InfiniteFocus Alicona system and CMM was used to validate the result. The twist springback was measured on a selected sections of the propeller blade. The primary contributions of this study are based on the following findings and observations:

- The deformation ratio has a significant effect on the twist springback. This assumption is evidenced by larger twist springback found in the tail of the blade

- The twist angle error depends on the thickness of the blade and the severity of the twist. A larger twist angle results in a larger error. Similarly, a thicker blade exhibits a more dominant error

- Optical images can provide a clear profile of the blade, which can be used to evaluate the geometric and dimensional defects

\section{ACKNOWLEDGEMENT}

The researchers want to acknowledge the Universiti Sains Malaysia for the Research University grant (PMEKANIK/814094).

\section{REFERENCES}

Abdullah, A.B., S.M. Sapuan, Z. Samad and N.A. Aziz, 2012a. A comprehensive review of experimental approaches used in the measurement of springback. Adv. Nat. Applied Sci., 6: 195-205.

Abdullah, A.B., S.M. Sapuan, Z. Samad, H.M.T. Khaleed and N.A. Aziz, 2012b. Geometrical error analysis of cold forged auv propeller blade using optical measurement method. Adv. Mater Res., 383390: 7117-7121.

Ambrogio, G., I. Costantino, L.D. Napoli, L. Filice and L. Fratini et al., 2004. Influence of some relevant process parameters on the dimensional accuracy in incremental forming: A numerical and experimental investigation. J. Mater Process Technol., 153-154: 501-507. DOI: 10.1016/j.jmatprotec.2004.04.139

Chang, Y.C., Z.M. Hu, B.S. Kang and T.A. Dean, 2002. A study of cold ironing as a post-process for netshape manufacture. Int. J. Mach. Tools Manuf., 42: 945-952. DOI: 10.1016/S0890-6955(02)00022-6

D'Acquisto, L. and L. Fratini, 2001. An optical technique for springback measurement in axisymmetrical deep drawing operations. J. Man Proc., 3: 29-29. DOI: 10.1016/S15266125(01)70031-X 
Dejardin, S., S. Thibaud, J.C. Gelin and G. Michel, 2010. Experimental investigations and numerical analysis for improving knowledge of incremental sheet forming process for sheet metal parts. J. Mater Proc. Technol., 210: 363-369. DOI: 10.1016/j.jmatprotec.2009.09.025

Habali, S.M. and I.A. Saleh, 2000. Local design, testing and manufacturing of small mixed airfoil wind turbine blades of glass fiber reinforced plastics Part I: Design of the blade and root. Energy Conv. Manage., 41: 249-280. DOI: 10.1016/S01968904(99)00103-X

Hsu, T.H., J.Y. Lai and W.D. Ueng, 2006. On the development of airfoil section inspection and analysis technique. Int. J. Adv. Manuf. Technol., 30: 129. DOI: $10.1007 / \mathrm{s} 00170-005-0046-5$

Lam, M.K., S.F. Lee, P. Iovenitti and S.H. Masood, 2002. A cost-effective thickness measurement technique for engine propellers. Int. J. Adv. Manuf. Technol., 20: 180-189. DOI: $10.1007 / \mathrm{s} 001700200141$

Lange, K., 1985. Handbook of Metal Forming. 1st Edn., McGraw-Hill, New York, ISBN-10: 0070362858, pp: 900.
Li, H., G. Sun, G. Li, Z. Gong and D. Liu et al., 2011. On twist springback in advanced high-strength steels. Mater. Des., 32: 3272-3279. DOI: 10.1016/j.matdes.2011.02.035

Li, X.B., H.W. Hu, Y. Yang, P.J. Ni and Y. Cheng, 2010. Automatic ultrasonic inspection of flaws in a propeller-blade. Nondestruct Test Eval., 25: 341351. DOI: $10.1080 / 10589759.2010 .489610$

Makem, J., H. Ou, C.G. Armstrong, A. Rennie and S. Nikov, 2008. A virtual inspection technique for assessing the dimensional accuracy of forged compressor blades using FE modeling and CMM inspection. Int. J. Metal Form., 1: 375-378. DOI: 10.1007/s12289-008-0073-5

Pahk, H.J. and W.J. Ahn, 1996. Precision inspection system for aircraft parts having very thin features based on CAD/CAI integration. Int. J. Adv. Manuf. Technol., 12: 442-449. DOI: 10.1007/BF01186933

Qiu, H., Li, K. Cheng, Y. Li and J. Wang, 2000. A study on an evaluation method for form deviations of 2D contours from coordinate measurement. Int. J. Adv. Manuf. Technol., 16: 413-423. DOI: $10.1007 / \mathrm{s} 001700050173$ 